\title{
Discussion on Applicability and Requirements for the Installation of Fastenings Using Robot Systems in the Construction Industry
}

\author{
Arnim Johannes Spengler ${ }^{1 *}$, Panagiotis Spyridis³ ${ }^{3}$ Tobias Bruckmann², Alexander Malkwitz \\ Dirk Schlüter ${ }^{1}$
}

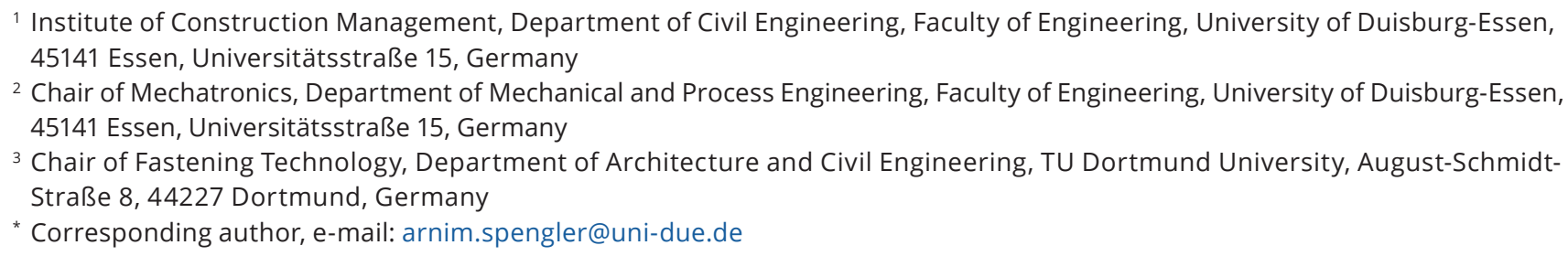

Received: 19 November 2019, Accepted: 31 March 2020, Published online: 28 May 2020

\begin{abstract}
Since robotics is becoming increasingly widespread in the construction industry, more phases and working steps should be investigated for their applicability to automation. Ideally, only few robot systems would be needed and thus be multifunctional.

Current robot systems are used almost exclusively in precast construction. At the construction site, only prototypes are in use, and only individual parts of the building shell construction and assembly can be handled.

This paper examines to what extent robots can be applied for the installation of fastenings and which boundary conditions exist or need to be addressed. Automated construction, and more precise installation of fastenings, has been partially implemented, which has been shown to increase productivity as well as installation quality, and therefore the components' structural safety. This knowledge must now be extended to robots. The present work is based on an overview of current research and development and includes a discussion on the current research at the University Duisburg-Essen on a cable robot for brickwork construction. It further demonstrates that fastenings pose an important additional application, especially to ensure the changeover to other building materials. These can be built-in parts, but also brickwork connections or prefabricated parts.

It can be assumed that robots will become increasingly important in the construction industry for reasons that include high quality, safety, speed and economic aspects.

This is an extended paper of the Creative Construction Conference in Budapest on "Examination of Advanced Fastening Systems for the use of Robots in the Construction Industry" (Spyridis et al., 2019).
\end{abstract}

\section{Keywords}

fixings, fastenings, construction robots, wire robots, automation in construction

\section{Introduction}

Robot systems in the construction industry have been investigated since the early 1980s. Since then, the use of robots was first established in factories for precast structural elements. The systems are used, as in other areas of stationary production, mainly for repetitive tasks. However, outside of this, robot systems have not yet been able to access the construction site, as discussed in previous publications (Bruckmann et al., 2016; Bruckmann et al., 2018; Cousineau and Miura, 1998).
Possible reasons for this are the reliance of the construction industry on 2D model prints, as well as unique project/product specifications, which create additional challenges when programming robots. Another reason is the limited working space of robots, i.e. lack of free movement systems for a robot over a large part of the construction site. However, these constraints seem to have experienced a period of transformation in recent years, while the assembly of printed construction elements was often applied (Vähä et al., 2013; Viscomi et al., 1994). 
This paper describes the requirements and possible methods in the construction industry for the installation of fixings and assembly of fitted elements by robots. Firstly, a brief outline of robotics in the construction industry is given. This is necessary to understand the open questions. On this basis, further research, potential and necessity are examined, and directions for further investigations are established. Following this, the importance of fixings in the described area of application is introduced. Then, some construction tasks and objectives with regards to fastenings are presented, with emphasis on those which a robot system can perform better than humans under specific conditions. Other reasons for considering the implementation of robots for these tasks are discussed, such as health and safety at work, quality, or avoidance of defects due to human error.

The use of robots in the construction industry and the discussion and fulfilment of the related requirements is a broad field. In this paper, robotic installations and assemblies are elaborated in the context of building components and the use of post-installed fastenings. Considered materials are sand-lime bricks, precast concrete elements and concrete poured on the construction site.

\section{Presentation of robot systems in the building industry}

In the 1980s, the topic of the automated construction of buildings was first discussed in the broader circle. At that time, the portal robot was considered to be particularly suitable for the construction of buildings. Today, these types of robots are often used because they can bridge large spans. Many of the earlier 2000 robot systems can be found in precast factories. Robot systems, which can be installed directly on the construction site, were not followed up beyond the conception phase. Some concepts considered an automated approach where the fixations are inserted from above and then covered with concrete. However, many do not address this topic at all, or these activities are still manually carried out. Successful examples are construction robots developed in Japan, where the reinforcement is interlaced manually. These activities will also continue to be performed manually for the systems presented in Table 1.

From a construction perspective, the manufacturing processes are particularly attractive. The systems shown in Table 1 can only be used to a limited extent in the building shell construction. Thus, the actual systems are limited to parts of the building shell and cannot be used for other construction methods. Consequently, many of the extended construction activities like the insertion of windows or setting the roof truss cannot currently be automated using these systems.
Table 1 Systems, basic platforms and manufacturing processes for (partially) autonomous building construction

\begin{tabular}{|c|c|c|}
\hline Name & Basis platform & manufacturing method \\
\hline Apis Cor & Modified robot arm & Contour Crafting \\
\hline Hadrian & $\begin{array}{l}\text { Modified crawler } \\
\text { excavator }\end{array}$ & brickwork \\
\hline Hadrian X & $\begin{array}{l}\text { Modified truck with } \\
\text { cantilever arm }\end{array}$ & brickwork \\
\hline SAM & Robot arm & brickwork \\
\hline $\begin{array}{l}\text { Wire robot for } \\
\text { construction }\end{array}$ & Wire robot & brickwork \\
\hline Spiderbot & Wire robot & Contour Crafting \\
\hline Kamermaker & Portal robot & Contour Crafting \\
\hline Vulcan + Vulcan II & Portal robot & Contour Crafting \\
\hline
\end{tabular}

When robot systems are to be used in the extended building shell and set fixings, new challenges arise:

- It is not possible to use the same mechanism for setting fastenings, for example, when building with concrete.

- The usage of multiple robot types is a cost driver. Which currently leads to a point of continuing with an existing robot system. Modular approaches can be more applicable if fastenings are optimized for robot use or robots are better able to set fastenings.

- It must be decided whether first the fixings and then the component should be set or both at the same time.

- Clarification is needed as to whether conventional or unconventional standardised fixing products can be used

- New fixing methods for use in robot-driven systems need to be standardised

- The construction process must remain continuous and undisturbed.

\section{Parameters that determine the use of fixtures by robot systems in the construction industry}

In addition to the challenges described, further parameters affect the use of robot systems. The following list provides an overview:

- Weight: Robot systems can be heavy, which makes it difficult to use them on site. For installation, the subfloor must be sufficiently load-bearing, installation on floor slabs is made more difficult or is not possible, and adequate stability must be demonstrated.

- Speed: The speed of the overall system for moving components has a direct influence on the overall construction time. This includes the procedure of moving the base platform, as well as the speed of the end 
effector (i.e. the tool carrier of the robot) and the tool itself.

- Complexity: The complexity is conditioned by properties such as operation, construction, programming and pre-processing.

- Payload: A low payload can be limiting. To achieve shorter construction times, the transportation of larger and thus heavier parts might be required. Some components cannot be made smaller or lighter and thus require a specific payload.

- Workspace: Not all working environment situations can be processed with all systems. Examples are working overhead or working in tight environments.

- Radius of action: A small radius of action (workspace) requires frequent repositioning of the robot system. Moving the entire system is at the expense of construction speed and might cause issues for precision.

- Height limitation: The systems should be able to erect several storeys without having to dismantle and erect them at great expense.

- Sensitivity to environmental influences: In order to be used on the construction site, sufficient insensitivity to environmental influences should be ensured. The systems should be insensitive to dust, heat, cold and water. Mechanical shocks are to be expected.

- Cost of use: The operational costs of the overall system have a direct influence on the economic efficiency and the operational hurdle for companies. New business models might be required.

In addition, it is likely that the use of robots only in shell construction is not economically efficient. The systems must be used in further construction phases. In these phases, the setting of fixtures becomes of essential importance. Work in the extended shell construction phase can include various fittings, such as the installation of facade elements, balconies, windows, door frames, HVAC parts, or receptors for building expansion. For these installations, advanced fastening systems, potentially with adaptable or generic assembly features, are important; consequently, robots can be used more efficiently by being involved with more Work Breakdown Structure (WBS) elements.

In the following, current conventional fixing types are described.

Modern fastening technology is characterised by a large variety of construction products (post-installed or cast-in-place fasteners), while the industry also supplies

purpose-produced fixtures for various building applications. Furthermore, a consistent set of product performance documents (EU Regulation No. 305/2011, 2011) and structural design calculation standards (NSAI, 2018) form the basis for specifying, detailing, and installing fastenings. The large variety of products in the market (see an exemplary presentation in Fig. 1) aims to satisfy a variety of performance requirements with considerations on minimising costs and assembly speeds. These requirements include available dimensional configurations, load-bearing capacities, and broader project specifications such as thermal and noise insulation, fire resistance, durability, environmental footprint, aesthetics, and ease of removal. Postinstalled anchors are mostly available for use in concrete and masonry, while some applications of post-installed anchors for steel structures, wood, and other construction materials are also available in the market. Although each anchor has specific installation procedures, the generic steps for anchor installation can be described as follows:

- Locating the fastening point: This can be done digitally in the CAD model of the project and can be geodetically verified onsite; automated location of the robot onsite is still subject to ongoing research, especially regarding costs (Vähä et al., 2013). Conflict checks or confirmation of the as-built reinforcement must also be

(a)

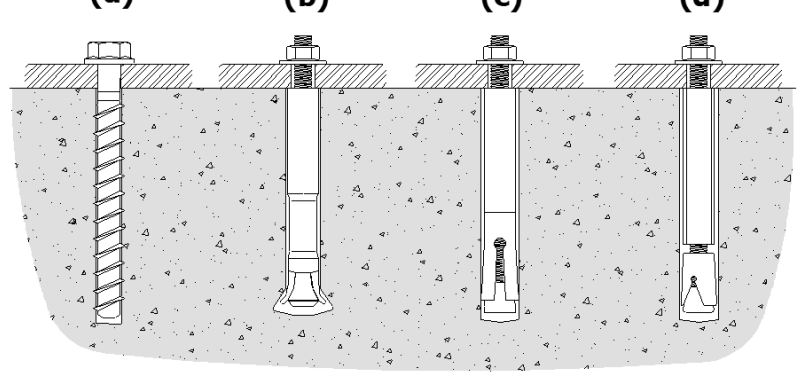

(e)

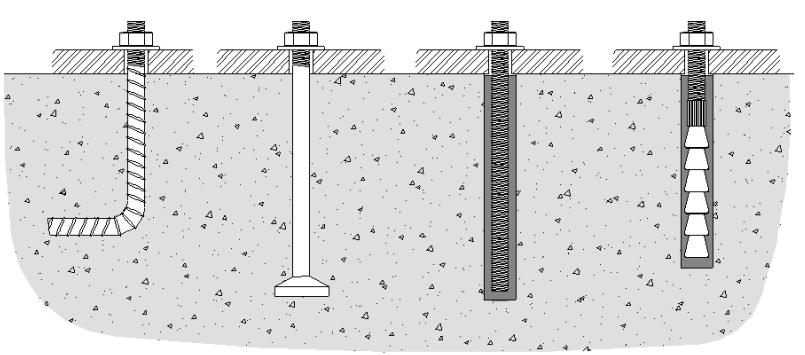

Fig. 1 Different anchor types, depending on the installation procedure: (a) concrete screw, (b) undercut anchor, (c) expansion anchor - sleeve type, (d) expansion anchor - bolt type, (e) cast-in-place ribbed and deformed bar, (f) cast-in-place headed stud, (g) bonded anchor with threaded rod, (h) special bonded anchor (all pre-positioned systems) 
performed in order to avoid rebar hits during the further installation steps. To that end, the real positioning of rebar and the conflict check can be performed based on a rebar detection system. The equipment needed in this step may include positioning system (and connected BIM software), marking tool, electromagnetic/ penetrating rebar radar.

- Borehole drilling: This requires that the drilling element is set perpendicularly $\left( \pm 3^{\circ}\right)$ to the substrate surface, and a drill hole is performed. This procedure can typically use either a hammer drill or a diamond core drill. In the latter case, the provision of water as a cooling and lubricant agent is needed. In both cases, the drilling procedure produces waste (either dust or contaminated water), which needs to be removed for health and environmental reasons. Recently, hollow drill elements have been developed that can withdraw any drilling waste internally through a vacuum suction tube. In case of post positioned anchors (see Fig. 2) a protective measure for the existing holes on the fixture needs to be envisaged, to protect both the drill bit and the fixture from friction with each other. The equipment needed in this step may include a positioning and angle system for the drill bit based on the system of the previous step, drilling, resistance force (self-weight in vertical applications), drill with hydraulic or compressor circuit, collection system for drill waste, and depth gauge. The dimensions of the drill bit must also be consistently checked to ensure that wearing of the drill bit has not exceeded the allowable limits.

- Cleaning of borehole: After finalising the drilling procedure, the drill dust must be removed for installation quality purposes. The means for this task are an air pump and steel brush when it comes to manual work. Hollow-core drill bits can also make up for this task.

- Preparation/mixing of injection mortar (for bonded anchors): This currently requires that the installation technician onsite visually checks if the

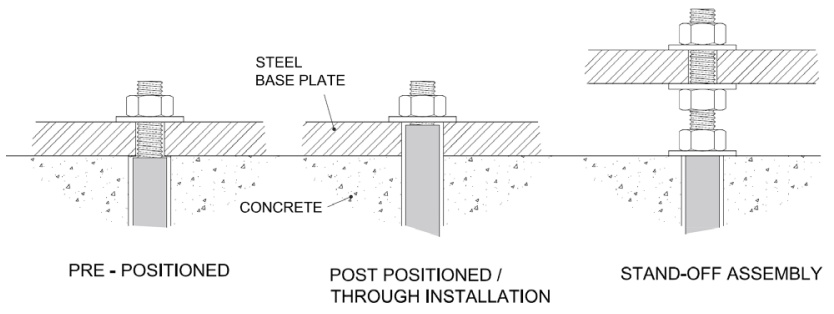

Fig. 2 Anchor installation configurations, dependent on logistic mortar's ingredients are uniformly mixed, based on the colour and texture of the mortar extracted from the injection nozzle. An alternative method for chemically bonded anchors is the capsule system, which can be mixed simultaneously with the injection of the anchor. The equipment needed for injection anchors may include an injection nozzle with an integrated flow sensor, connected to a two-component container with the bonding chemicals. For the capsule system, only a rotational tool for the insertion and drilling of an anchor-capsule system is necessary. It is also notable that capsule anchors are less sensitive to the quality of the borehole cleaning.

- Collection/Selection and insertion of the anchor at appropriate depth: To this end, either a predefined feed of anchors should be in place, or the robotic system must identify and pick the correct anchoring insert. The anchors should be equipped by a marker showing the type, dimensions, and orientation of the insert, while a visual identifier must collect and insert the anchor. For an unstructured feed of anchors to the installation robot, a generic visual identifying software via a camera must be in place. Alternatively, a cassette with predefined positions of the fasteners must be available. An automated collection grip or magnetic tip is necessary for the selection and insertion of the anchor.

- Tightening of fastener: When tightening the end of the anchor, may this be a nut or screw end type, the correct torque must be applied. Currently used rotation tools are typically equipped with torque monitor devices, set to immediately end the application of torque once a specific torque level is reached. Such equipment should also be provided in a robotic system.

- Load-testing of anchor: Once an anchor is installed, an installation check or even proof testing before commissioning the project is often required, specifically for projects of high significance (tunnels or nuclear plants). This can be done by applying a load on the anchor (a multiple of the characteristic load) and observing the anchor's displacement behaviour. In all cases, an optical check can also provide some confidence for the appropriate installation of the anchor. Additional equipment for this task can be a photographic camera and a hydraulic or electric pulling frame, which must again be linked automatically to the anchor head or the installed fixture. 
Furthermore, mostly depending on the nature of the fixture, and the construction plan, two different types of fixture assembly can be used as seen in Fig. 2. The assembly with pre-positioned anchors requires that all the above steps up to anchor installation occur before placing and securing the fixture. This requires that the drilled hole in the base material is larger than the clearance hole in the fastened element. Simultaneously, this requires exact positioning of the anchor in order to avoid clashes with the fixture holes. Postinstallation configurations use the element to be fastened as a drilling and setting template for the anchors. In this case, the diameter of the hole in the component to be fastened should be larger than or equal to the borehole diameter. In this case, it is also important to provide for safe temporary support of the fixture during the anchor setting procedure. A practical method - though not always applicable - is mixing the above methods by temporarily supporting the fixture with one pre-positioned anchor and subsequently installing the rest of the anchors. An issue with post-installation is the possible damage of the fixture due to the through-drilling procedures, which compromises the appearance and the durability of the system.

\section{Requirements for advanced fastening systems}

Prefabrication, automated erection, and modularisation procedures gain in relevance hand in hand with the increasing implementation of digital construction technologies. Besides the parameters of robotised construction discussed above, the use of automated procedures can generally minimise or eliminate typical health and safety risks related to the installation of fixings. These include hand injuries due to drill torque reaction, hand-arm vibrations, inhaling of dust, high noise levels, working with chemicals, electric shocks. Robotised construction can also facilitate the installation of fixings where human presence is of unacceptably high health and safety risks or virtually impossible. These cases include installations in very confined spaces, work at height, underwater installations, construction at extreme environmental or space conditions, rehabilitation or decommissioning of industrial and nuclear facilities.

Moreover, it has been widely proven that human errors in the installation of fixings can substantially decrease the load-bearing capacity of the anchor, occasionally even down to $20 \%$ of the expected resistance. Simultaneously, statistics and industry reviews have shown that many construction professionals may not be aware of the exact installation procedures for fixings (Cronin, 2015; Grosser et al., 2011).
A faulty installation can not only lead to reduced structural safety at the beginning of the fastenings life-cycle, but defects at installation can also propagate to faster degradation of the fixing and possibly a failure without prior warning (CIRIA, 2019). These facts undoubtedly lead to very high technical risks, and this has already led to catastrophic collapses in the past. In order to ensure quality of construction and a permanently high safety level, robotised construction, where human quality control is assisted by automated procedures, should be considered.

It should also be mentioned that current design codes propose an additional design safety factor for fixings on the load-bearing capacity to account for the installation quality (fib, 2011). This safety factor increases the design demand by $40 \%$ for less than normal installation safety (e.g. untrained or inexperienced personnel). This also shows a directly proportional increase as regards all aspects of fixings installation, such as procurement cost, time, and material resources.

As regards positioning of the anchor, an often-recurring problem is drilling onto or through rebar in concrete. In this case, both the function of the anchor and the efficiency of the reinforcement can be compromised; as such, the borehole is cancelled, and a new one must be drilled, at a minimum distance of a few centimetres. This means that the fixing point must also be redesigned. In some cases, this is also due to over or under-drilling. In order to avoid this, the as-built drawings, and a ferromagnetic rebar scanner are employed. Tolerances also play a vital role in fastening technology, as they can be decisive for the success of the assembly.

Furthermore, tolerances can strongly influence the distribution of loads from the fixture to the individual anchors, such as in a group, and consequently, lead to excessive load on a single anchor. In the absence of redundancy measures, failure of a single anchor can even lead to the collapse of the entire fixture (Spyridis and Bergmeister, 2012). The requirements for the fixing system used for an automated assembly are derived from the above descriptions. In summary, specific rules and methods need to be set as regards the interfaces and integration of fastening products with existing robot systems. Also, the generic steps for anchor installation must be reproducible, and insensitive to the location, or variabilities as regards the base material, fixture, or intended use. The fixing product should comply with existing standards, while it should be noted that these standards (typically the certified manufacturer's declaration of performance) dictate the construction/installation method. Finally, it should provide the logistical benefits in order to not negatively affect the speed and construction sequence of the system. 
An ideal application would allow the robot system to immediately integrate the complete fastened system (i.e. fixing and fixed component) into the building. This requires a fixing method that accommodates it from a load-bearing and a logistic viewpoint. If the fixing installation is nonetheless performed manually, a robot system should be able to control whether they have been made correctly, by onsite testing and evaluation.

It is noteworthy that the application of robot systems might require the use of marked construction elements, each having a unique identity (ID) such as a QR code or NFC tag. This creates significant potential for pre-installation of elements like anchors. This concept needs further investigation.

\section{Effects on the robot system}

The task of setting fixings on the construction site is difficult to implement for robot systems generally. Fig. 3 shows theoretically reachable positions for a robot arm and a wire robot.

It shows that the robot arm covers more positions of a building already erected with the disadvantage that the robot must be moved to be able to cover a larger area. Additionally, its range is limited by its radius and the fact that the mobile platform can only move on solid ground. After any movement, the platform needs to be localised in space. The wire robot can only work at the height of the current construction level, but the workspace is much larger. For setting fixations, this means that both systems can be used differently. Advantages of individual systems, such as the large working area, can be disadvantageous in other tasks. For example, working in confined spaces, closed rooms, or when the ground is not sufficiently load-bearing.

The procedures for setting fixations have a significant influence on practical usability. All work steps require different manipulation devices, so process steps must be optimized. At this time, it is not possible to recommend an optimal process design; this will be investigated in further studies. However, it can be assumed that every robot system has its own optimal workflow. Table 2 gives a pro

Robot arm

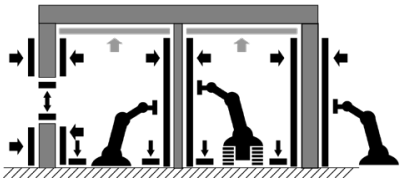

Fig. 3 Theoretically reachable positions using a robot arm and wire robot, respectively. The wire robot an only act at the current height level of the building.

and contra overview of the exemplary drilling process step.

It has been shown that the robot arm covers more positions of an already erected building with the disadvantage that the robot must be moved to be able to cover a larger area. Additionally, its range is limited by its radius and that the mobile platform can only move on solid ground. After any movement, the platform needs to be localised in space. The wire robot can only work at the height of the current construction level, although, the workspace is much larger. For setting fixations, this means that both systems can be used differently. Advantages of individual systems, such as the large working area, can be

Table 2 Pro and contra overview of the exemplary process step drilling

\begin{tabular}{lll}
\hline Robot arms & $\begin{array}{l}\text { Robot arms on a } \\
\text { mobile platform }\end{array}$ & Wire robot systems \\
&
\end{tabular}

pro: Robot arms can reach hard-toreach positions and have high degrees of flexibility.

contra: The workspace is comparatively small. The robot must be repositioned to reach poses [a pose is the combination of position and orientation in space] outside its current workspace.

pro: Overhead drilling possible

contra: Robot arms can only manipulate their direct periphery. If they are fixed, then they can apply pressure. The robot must be repositioned to reach poses outside its current workspace. pro: Advantages of the single robot arm combined with an extension of the translationa workspace in the horizontal plane

contra: High effort when using the rails; high demands on the planeness of the rails. Mobile platforms on wheels or caterpillar drives need advanced navigation and localization

pro: Advantages of the single robot arm combined with a larger reachable area.

contra: High effort when laying the rails. High demands on the planeness of the rails. pro: Wire robot can drive over a large area and drill all necessary holes in the horizonta plane without repositioning of the support structure.

contra: Drilling force is limited. For high precision, pose measurement is required.

pro: in the area in which the manipulator can work, all drilling operations in the current height

level can be carried out without repositioning of the support structure.

contra: Work can only be carried out to a limited extent within the enclosing area of a building structure. 
disadvantageous in other tasks. An example of this would be working in confined spaces, closed rooms, or when the ground is not sufficiently load-bearing.

The procedures for setting fixations have a significant influence on the practical usability. All work steps require different manipulation devices; consequently, the process steps must be optimised. At this time, it is not possible to recommend an optimal process design; this should be investigated in further studies where even paradigm changes might be necessary (e.g. Robot-Oriented Design) (Bock, 1988). However, it can be assumed that every robot system has its own optimal workflow. Table 2 gives a pro and contra overview of the exemplary drilling process step.

In order to minimize tool changes on the robot, holes can be pre-drilled at the factory. This procedure is only possible for prefabricated parts and bricks and requires a unique identification (ID) of the parts to be installed.

\section{Conclusion}

This study addresses the use of robots in construction. It departs from extended phases of brickwork or contour crafting, toward the potential and efficiency that can be achieved when robotic installations are developed for fastenings and assemblies of fitted components in construction. This further potential of robotised construction applications is discussed based on particularities and benefits of robotic construction, current obstacles and risks in manual fastening installations, and how these two aspects of construction can be integrated toward a safe and efficient practice.

\section{References}

Bock, T. A. (1988) "Robot-Oriented Design", In: The 5th International Symposium on Automation and Robotics in Construction (ISARC 1988), Tokyo, Japan, pp. 135-144. https://doi.org/10.22260/ISARC1988/0019

Bruckmann, T., Mattern, H., Spengler, A., Reichert, C., Malkwitz, A., König, M. (2016) "Automated Construction of Masonry Buildings using Cable-Driven Parallel Robots", In: 33rd International Symposium on Automation and Robotics in Construction (ISARC 2016), Auburn, AL, USA, pp. 332-340. https://doi.org/10.22260/ISARC2016/0041

Bruckmann, T., Reichert, C., Meik, M., Lemmen, P., Spengler, A., Mattern, H., König, M. (2018) "Concept Studies of Automated Construction Using Cable-Driven Parallel Robots", In: Gosselin, C., Cardou, P., Bruckmann, T., Pott, A. (eds.) Cable-Driven Parallel Robots, Springer, Cham, Switzerland, pp. 364-375. https://doi.org/10.1007/978-3-319-61431-1_31

Construction Industry Research and Information Association (2019) "General fixings Guidance on selection and whole life management C777", Construction Industry Research and Information Association (CIRIA), London, UK.
Based on this exercise, the efficiency of automated fastening assemblies is discerned, while important new research questions arise. These questions also pose the challenges for research investigations and the development of efficient technologies. As regards the first applications of robotic systems, the University of Duisburg-Essen is currently developing a cable robot to investigate automated brickwork construction (Malkwitz, 2019). The scientific teams involved envisage using this robot system to address the particularities of fastening applications for specific building systems, with appropriate adjustments and extensions of the present system. To this end, to provide suitable systems, further research is necessary along the lines of the open questions outlined below:

- Which robot types and end effector types are potentially usable for the installation of fastenings and fitted components?

- In which construction phases can automated fixation solutions be used?

- Why do current robot systems not set fixations in the construction industry?

- How do other industries install automated fastenings?

- Which design and construction standards are involved, and how should these be adapted?

Once these research questions are addressed, a knowledge base will become available in order to translate the integrated automated fastening assembly into a real construction system.

Cousineau, L., Miura, N. (1998) "Construction robots: the search for new building technology in Japan", ASCE Publications, Reston, VA, USA.

Cronin, B. (2015) "Industry fears over fixings", New Civil Engineer, [online] 10 September 2015. Available at: https://www.newcivilengineer.com/latest/industry-fears-over-fixings-10-09-2015/ [Accessed: 02 November 2019]

European Parliament and Council (2011) "EU Regulation No. 305/2011 Laying down harmonised conditions for the marketing of construction products and repealing Council Directive 89/106/EEC", Construction Products Regulation (CPR), Brussels, Belgium.

Grosser, P., Fuchs, W., Eligehausen, R. (2011) "A Field Study of Adhesive Anchor Installations", Concrete International, 33(1), pp. 57-63.

Malkwitz, A., Spengler, A. J., Bruckmann, T. (2019) "Baubetriebliche Untersuchung von Robotersystemen im Mauerwerksbau" (Operational Investigation of Robot Systems in Masonry Construction), Bautechnik, 96(5), pp. 375-379. (in German) https://doi.org/10.1002/bate.201800090

Spyridis, P., Bergmeister, K. (2012) "Effects of Assembly Tolerances on Bolted Anchorages in Concrete", Journal of Structural Engineering, 140(1), Article Number: 04013024. https://doi.org/10.1061/(ASCE)ST.1943-541X.0000776 
Spyridis, P., Spengler, A. J., Schlüter, D., Bruckmann, T., Malkwitz, A. (2019) "Examination of Advanced Fastening Systems for the use of Robots in the construction industry", In: Creative Construction Conference (CCC 2019), Budapest, Hungary, pp. 323-328. https://doi.org/10.3311/CCC2019-046

The International Federation for Structural Concrete (2011) "Design of anchorages in concrete", The International Federation for Structural Concrete (fib), Lausanne, Switzerland.

The National Standards Authority of Ireland (2018) "EN 1992-4:2018. Eurocode 2: Design of concrete structures - Part 4: Design of fastenings for use in concrete", The National Standards Authority of Ireland (NSAI), Dublin, Ireland.
Vähä, P., Heikkilä, T., Kilpeläinen, P., Järviluoma, M., Gambao, E. (2013) "Extending automation of building construction - Survey on potential sensor technologies and robotic applications", Automation in Construction, 36, pp. 168-178.

https://doi.org/10.1016/j.autcon.2013.08.002

Viscomi, B. V., Michalerya, W. D., Lu, L. W. (1994) "Automated construction in the ATLSS integrated building systems", Automation in Construction, 3(1), pp. 35-43.

https://doi.org/10.1016/0926-5805(94)90030-2 